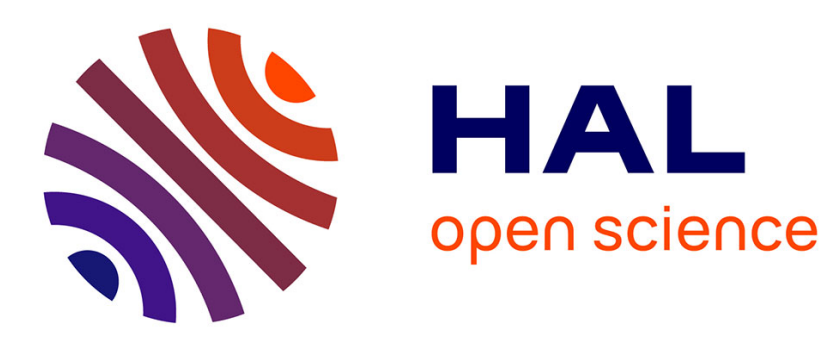

\title{
Benchmarking the Pure Random Search on the BBOB-2009 Noisy Testbed
}

\author{
Anne Auger, Raymond Ros
}

\section{To cite this version:}

Anne Auger, Raymond Ros. Benchmarking the Pure Random Search on the BBOB-2009 Noisy Testbed. ACM-GECCO Genetic and Evolutionary Computation Conference, Jul 2009, Montreal, Canada. inria-00430534

\section{HAL Id: inria-00430534 https://hal.inria.fr/inria-00430534}

Submitted on 8 Nov 2009

HAL is a multi-disciplinary open access archive for the deposit and dissemination of scientific research documents, whether they are published or not. The documents may come from teaching and research institutions in France or abroad, or from public or private research centers.
L'archive ouverte pluridisciplinaire HAL, est destinée au dépôt et à la diffusion de documents scientifiques de niveau recherche, publiés ou non, émanant des établissements d'enseignement et de recherche français ou étrangers, des laboratoires publics ou privés. 


\section{Benchmarking the Pure Random Search on the BBOB-2009 Noisy Testbed}

\author{
Anne Auger \\ TAO Team - INRIA Saclay \\ LRI, Bat 490, Univ. Paris-Sud \\ 91405 Orsay Cedex, France \\ anne.auger@inria.fr
}

\author{
Raymond Ros \\ Univ. Paris-Sud, LRI \\ UMR 8623 / INRIA Saclay, projet TAO \\ F-91405 Orsay, France \\ raymond.ros@Iri.fr
}

\begin{abstract}
We benchmark the Pure-Random-Search algorithm on the BBOB 2009 noisy testbed. Each candidate solution is sampled uniformly in $[-5,5]^{D}$, where $D$ denotes the search space dimension. The maximum number of function evaluations chosen is $10^{6}$ times the search space dimension. With this budget the algorithm is not able to solve any single function of the testbed.
\end{abstract}

\section{Categories and Subject Descriptors}

G.1.6 [Numerical Analysis]: Optimization, Global Optimization, Unconstrained Optimization; F.2.1 [Analysis of Algorithms and Problem Complexity]: Numerical Algorithms and Problems

\section{General Terms}

Algorithms

\section{Keywords}

Benchmarking, Black-box optimization, Evolutionary computation, Pure random search, Monte-Carlo

\section{INTRODUCTION}

The pure random search proposed by Brooks in 1958 [2] is the most simple stochastic search algorithm. It consists in sampling each search point independently in the search domain and keeping the best solution found.

In this paper, we benchmark the pure random search where each solution is sampled uniformly in $[-5,5]^{D}$, where $D$ denotes the dimension of the search space. The maximum number of function evaluations is fixed to $10^{6} \times D$. The CPU time experiments and implementation details are given in the companion paper [1].

\section{RESULTS AND DISCUSSION}

Results from experiments according to [4] on the benchmarks functions given in $[3,5]$ are presented in Figures 1 and 2 and in Tables 1 and 2 .

Not too surprisingly, the pure random search cannot solve any function of the testbed. However, the results provide reference results useful for the investigation of more advanced algorithms.

\section{Acknowledgments}

We would like to thank Nikolaus Hansen for the way he led the BBOB project, Steffen Finck and Nikolaus Hansen for their great and hard work. We also would like to thank Marc Schoenauer for his kind support and essential help on the $\mathrm{C}$-code.

\section{REFERENCES}

[1] A. Auger and R. Ros. Benchmarking the Pure Random Search on the BBOB-2009 Testbed. In Workshop Proceedings of the GECCO Genetic and Evolutionary Computation Conference. ACM, 2009.

[2] S. H. Brooks. A discussion of random methods for seeking maxima. Operations Research, 6:244- 251, 1958.

[3] S. Finck, N. Hansen, R. Ros, and A. Auger. Real-parameter black-box optimization benchmarking 2009: Presentation of the noisy functions. Technical Report 2009/21, Research Center PPE, 2009.

[4] N. Hansen, A. Auger, S. Finck, and R. Ros. Real-parameter black-box optimization benchmarking 2009: Experimental setup. Technical Report RR-6828, INRIA, 2009.

[5] N. Hansen, S. Finck, R. Ros, and A. Auger. Real-parameter black-box optimization benchmarking 2009: Noisy functions definitions. Technical Report RR-6869, INRIA, 2009.
Permission to make digital or hard copies of all or part of this work for personal or classroom use is granted without fee provided that copies are not made or distributed for profit or commercial advantage and that copies bear this notice and the full citation on the first page. To copy otherwise, to republish, to post on servers or to redistribute to lists, requires prior specific permission and/or a fee.

GECCO'09, July 8-12, 2009, Montréal Québec, Canada.

Copyright 2009 ACM 978-1-60558-505-5/09/07 ...\$5.00. 

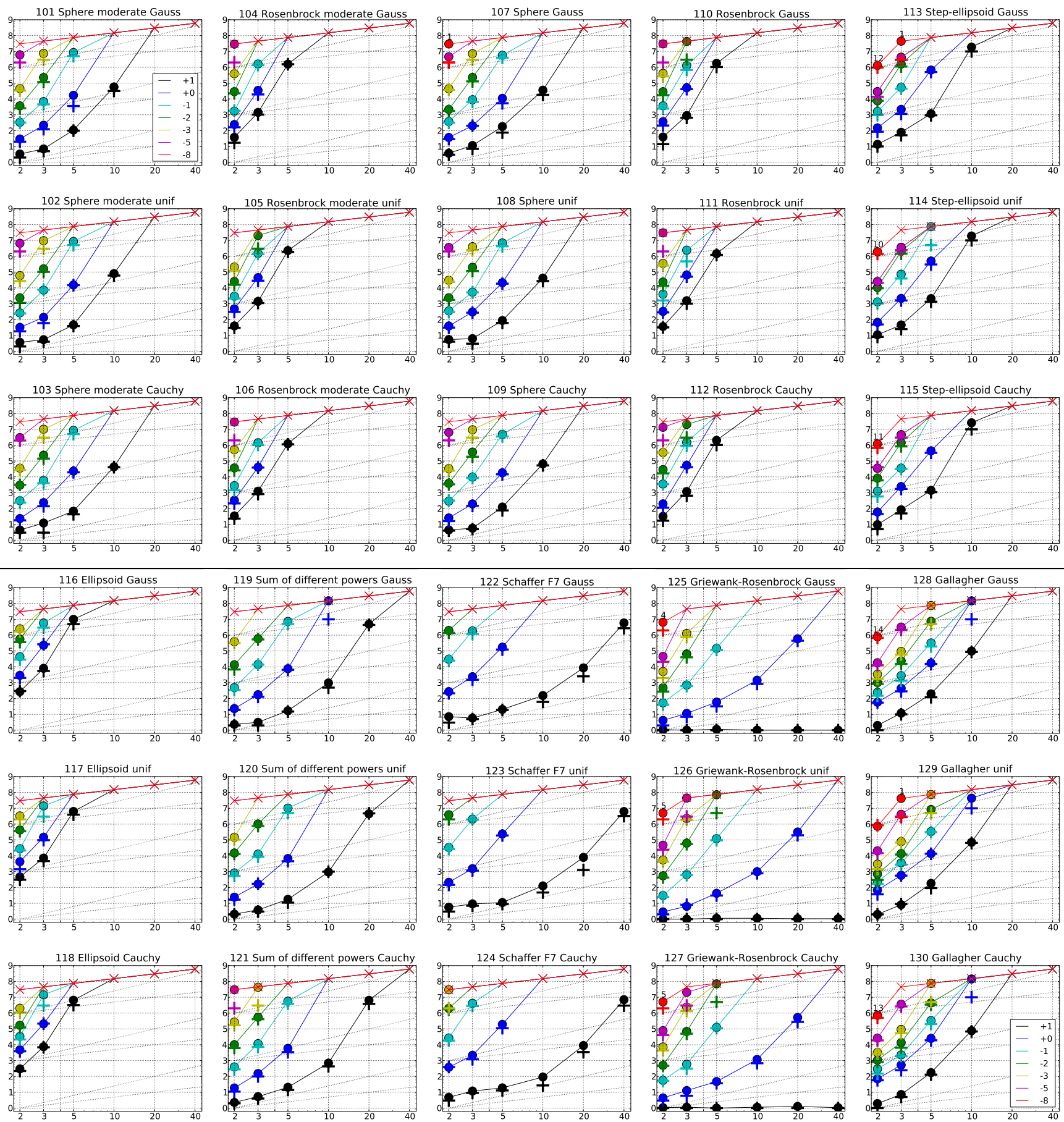

Figure 1: Expected Running Time (ERT, @) to reach $f_{\mathrm{opt}}+\Delta f$ and median number of function evaluations of successful trials $(+)$, shown for $\Delta f=10,1,10^{-1}, 10^{-2}, 10^{-3}, 10^{-5}, 10^{-8}$ (the exponent is given in the legend of $f_{101}$ and $\left.f_{130}\right)$ versus dimension in log-log presentation. The $\operatorname{ERT}(\Delta f)$ equals to \#FEs $(\Delta f)$ divided by the number of successful trials, where a trial is successful if $f_{\mathrm{opt}}+\Delta f$ was surpassed during the trial. The \#FEs $(\Delta f)$ are the total number of function evaluations while $f_{\mathrm{opt}}+\Delta f$ was not surpassed during the trial from all respective trials (successful and unsuccessful), and $f_{\text {opt }}$ denotes the optimal function value. Crosses $(\times)$ indicate the total number of function evaluations \#FEs $(-\infty)$. Numbers above ERT-symbols indicate the number of successful trials. Annotated numbers on the ordinate are decimal logarithms. Additional grid lines show linear and quadratic scaling. 


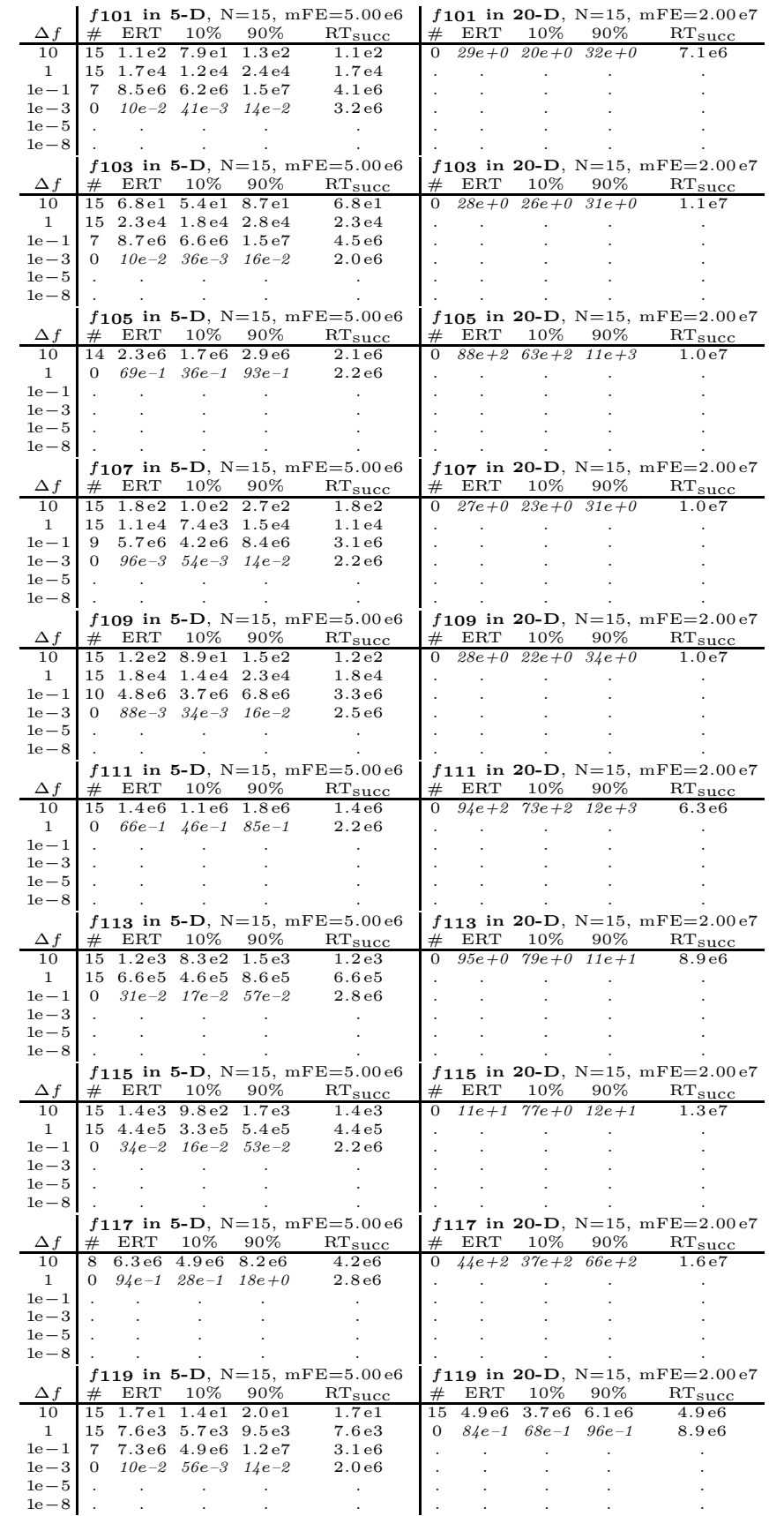



Table 1: Shown are, for functions $f_{101}-f_{120}$ and for a given target difference to the optimal function value $\Delta f$ : the number of successful trials (\#); the expected running time to surpass $f_{\mathrm{opt}}+\Delta f$ (ERT, see Figure 1); the $10 \%$-tile and $90 \%$-tile of the bootstrap distribution of ERT; the average number of function evaluations in successful trials or, if none was successful, as last entry the median number of function evaluations to reach the best function value $\left(\mathbf{R T}_{\text {succ }}\right)$. If $f_{\mathrm{opt}}+\Delta f$ was never reached, figures in italics denote the best achieved $\Delta f$-value of the median trial and the $10 \%$ and $90 \%$-tile trial. Furthermore, $\mathbf{N}$ denotes the number of trials, and $\mathrm{mFE}$ denotes the maximum of number of function evaluations executed in one trial. See Figure 1 for the names of functions. 

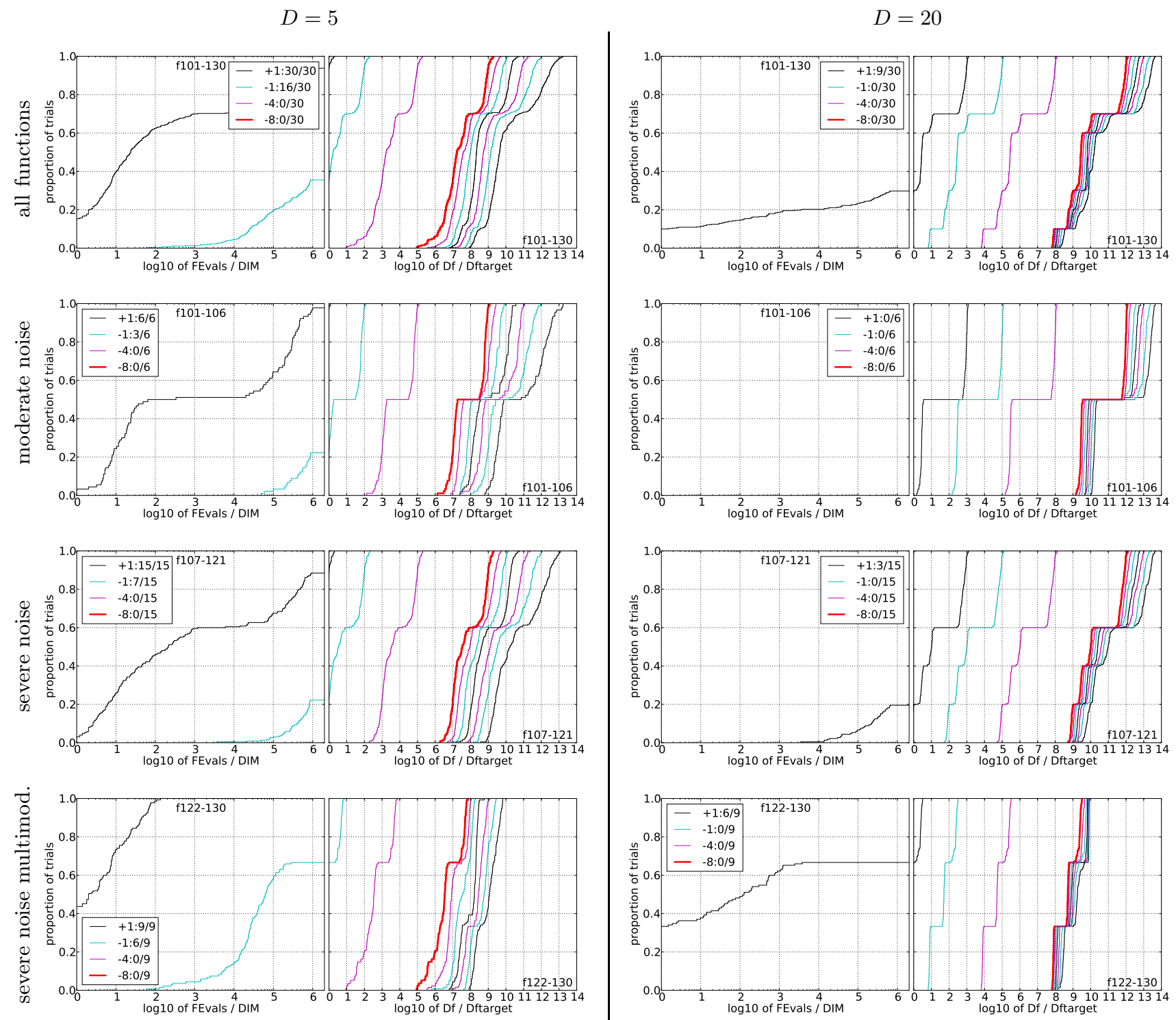

Figure 2: Empirical cumulative distribution functions (ECDFs), plotting the fraction of trials versus running time (left subplots) or versus $\Delta f$ (right subplots). The thick red line represents the best achieved results. Left subplots: ECDF of the running time (number of function evaluations), divided by search space dimension $D$, to fall below $f_{\text {opt }}+\Delta f$ with $\Delta f=10^{k}$, where $k$ is the first value in the legend. Right subplots: ECDF of the best achieved $\Delta f$ divided by $10^{k}$ (upper left lines in continuation of the left subplot), and best achieved $\Delta f$ divided by $10^{-8}$ for running times of $D, 10 D, 100 \mathrm{D} \ldots$ function evaluations (from right to left cycling blackcyan-magenta). Top row: all results from all functions; second row: moderate noise functions; third row: severe noise functions; fourth row: severe noise and highly-multimodal functions. The legends indicate the number of functions that were solved in at least one trial. FEvals denotes number of function evaluations, $D$ and DIM denote search space dimension, and $\Delta f$ and Df denote the difference to the optimal function value. 


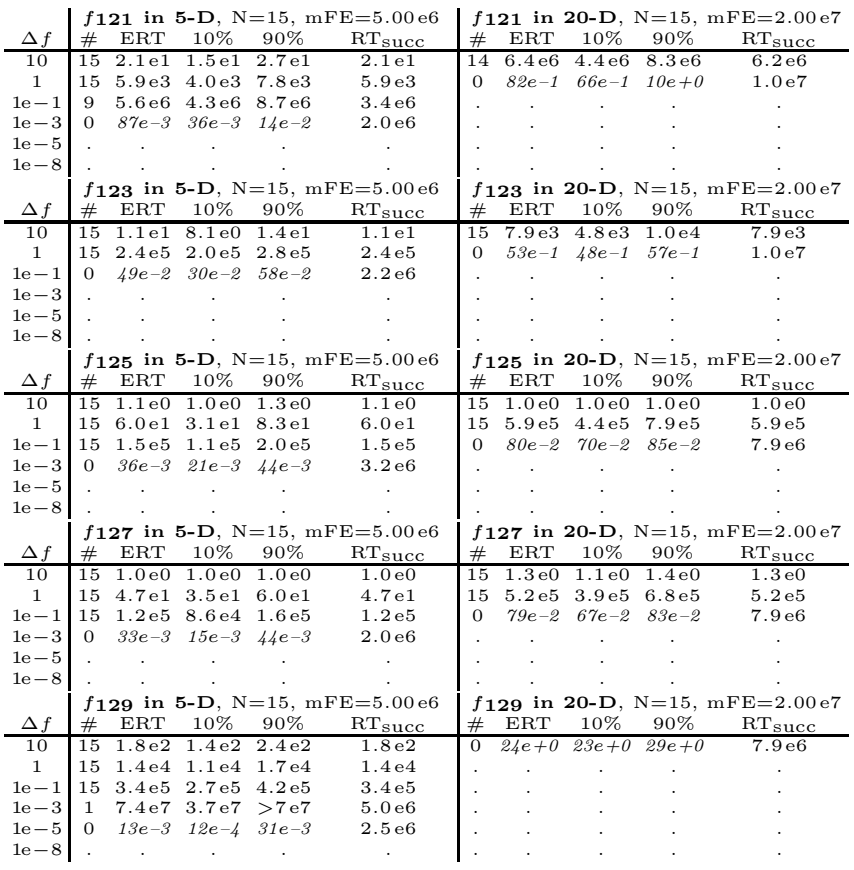

\begin{tabular}{|c|c|c|c|c|c|c|c|c|c|c|}
\hline \multirow[b]{2}{*}{$\Delta f$} & \multicolumn{2}{|c|}{$f_{122}$ in } & \multirow{2}{*}{\multicolumn{3}{|c|}{$5-\mathrm{D}, \mathrm{N}=15, \mathrm{mFE}=5.00 \mathrm{e} 6$}} & \multicolumn{5}{|c|}{$f 122$ in $20-\mathrm{D}, \mathrm{N}=15, \mathrm{mFE}=2.00 \mathrm{e}$} \\
\hline & \# & ERT & & & & \# & ERT & $10 \%$ & $90 \%$ & $\mathrm{RT}_{\mathrm{succ}}$ \\
\hline 10 & 15 & $2.0 \mathrm{e} 1$ & $1.4 \mathrm{e} 1$ & $2.6 \mathrm{e} 1$ & $2.0 \mathrm{e} 1$ & 15 & $8.6 \mathrm{e} 3$ & $4.8 \mathrm{e} 3$ & $1.3 \mathrm{e} 4$ & $8.6 \mathrm{e} 3$ \\
\hline 1 & 15 & $1.8 \mathrm{e} 5$ & $1.4 \mathrm{e} 5$ & $2.3 \mathrm{e} 5$ & $1.8 \mathrm{e} 5$ & 0 & $49 e-1$ & $44 e-1$ & $56 e-1$ & $1.0 \mathrm{e} 7$ \\
\hline $1 e-1$ & 0 & $46 e-2$ & $31 e-2$ & $56 e-2$ & $2.2 \mathrm{e} 6$ & & 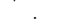 & . & & \\
\hline $1 e-3$ & & . & & & & & . & 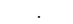 & . & . \\
\hline $1 e-5$ & & 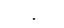 & . & & & & . & . & & \\
\hline \multirow{2}{*}{$1 e-8$} & . & . & . & . & & & . & 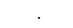 & . & . \\
\hline & \multicolumn{5}{|c|}{$f 124$ in $5-\mathrm{D}, \mathrm{N}=15, \mathrm{mFE}=5.00 \mathrm{e} 6$} & \multicolumn{5}{|c|}{$f 124$ in $20-\mathrm{D}, \mathrm{N}=15, \mathrm{mFE}=2.00 \mathrm{e} 7$} \\
\hline$\Delta f$ & \# & ERT & $10 \%$ & $90 \%$ & $\mathrm{RT}_{\text {succ }}$ & \# & ERT & $10 \%$ & $90 \%$ & $\mathrm{RT}_{\text {succ }}$ \\
\hline$\frac{10}{10}$ & $\frac{\pi}{15}$ & $1.9 \mathrm{e} 1$ & $1.4 \mathrm{e} 1$ & $2.3 \mathrm{e} 1$ & $1.9 \mathrm{e} 1$ & $\frac{\pi}{15}$ & $8.9 \mathrm{e} 3$ & $3.7 \mathrm{e} 3$ & $1.5 \mathrm{e} 4$ & $8.9 \mathrm{e} 3$ \\
\hline 1 & 15 & $1.9 \mathrm{e} 5$ & $1.2 \mathrm{e} 5$ & $2.7 \mathrm{e} 5$ & $1.9 \mathrm{e} 5$ & 0 & $51 e^{-1}$ & $48 e-1$ & $57 e-1$ & $1.1 \mathrm{e} 7$ \\
\hline $1 e-1$ & 0 & $46 e-2$ & $25 e-2$ & $61 e-2$ & $2.5 \mathrm{e} 6$ & & . & . & 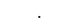 & 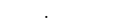 \\
\hline $1 e-3$ & . & . & & . & . & . & . & . & . & . \\
\hline $1 e-5$ & & . & . & & & & & . & . & . \\
\hline \multirow[t]{2}{*}{$1 \mathrm{e}-8$} & & & & & & & & & & \\
\hline & \multirow{2}{*}{\multicolumn{5}{|c|}{$f 126$ in $5-\mathrm{D}, \mathrm{N}=15, \mathrm{mFE}=5.00 \mathrm{e} 6$}} & \multicolumn{5}{|c|}{$f 126$ in $20-\mathrm{D}, \mathrm{N}=15, \mathrm{mFE}=2.00 \mathrm{e} 7$} \\
\hline$\Delta f$ & \# & ERT & & $90 \%$ & $\mathrm{RT}_{\text {succ }}$ & \# & ERT & $10 \%$ & $90 \%$ & $\mathrm{RT}_{\text {succ }}$ \\
\hline 10 & 15 & $1.1 \mathrm{e} 0$ & $1.0 \mathrm{e} 0$ & $1.3 \mathrm{e} 0$ & $1.1 \mathrm{e} 0$ & 15 & $1.1 \mathrm{e} 0$ & $1.0 \mathrm{e} 0$ & $1.1 \mathrm{e} 0$ & $1.1 \mathrm{e} 0$ \\
\hline 1 & 15 & $4.3 \mathrm{e} 1$ & $2.8 \mathrm{e} 1$ & $5.4 \mathrm{e} 1$ & $4.3 \mathrm{e} 1$ & 15 & $3.2 \mathrm{e} 5$ & $2.0 \mathrm{e} 5$ & $4.3 \mathrm{e} 5$ & $3.2 \mathrm{e} 5$ \\
\hline $1 e-1$ & 15 & $1.2 \mathrm{e} 5$ & $9.6 \mathrm{e} 4$ & $1.5 \mathrm{e} 5$ & $1.2 \mathrm{e} 5$ & 0 & $76 e-2$ & $72 e-2$ & $81 e-2$ & $8.9 \mathrm{e} 6$ \\
\hline $1 \mathrm{e}-3$ & 0 & $34 e-3$ & $24 e-3$ & $51 e-3$ & $1.4 \mathrm{e} 6$ & & . & . & (0) & 8.000 \\
\hline $1 e-5$ & . & 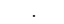 & . & 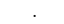 & . & . & . & . & . & . \\
\hline \multirow[t]{2}{*}{$1 e-8$} & & . & & & & & & & & \\
\hline & \multirow{2}{*}{\multicolumn{5}{|c|}{$f 128$ in $5-\mathrm{D}, \mathrm{N}=15, \mathrm{mFE}=5.00 \mathrm{e} 6$}} & \multicolumn{5}{|c|}{$f 128$ in $20-\mathrm{D}, \mathrm{N}=15, \mathrm{mFE}=2.00 \mathrm{e} 7$} \\
\hline$\Delta f$ & \# & & & & $\mathrm{RT}_{\mathrm{succ}}$ & \# & ERT & $10 \%$ & $90 \%$ & $\mathrm{RT}_{\text {succ }}$ \\
\hline 10 & 15 & $2.0 \mathrm{e} 2$ & $1.4 \mathrm{e} 2$ & $2.5 \mathrm{e} 2$ & $2.0 \mathrm{e} 2$ & 0 & $24 e+0$ & $15 e+0$ & $31 e+0$ & $1.0 \mathrm{e} 7$ \\
\hline & 15 & $1.7 \mathrm{e} 4$ & $1.4 \mathrm{e} 4$ & $2.1 \mathrm{e} 4$ & $1.7 \mathrm{e} 4$ & & , & , & . & . \\
\hline $1 e-1$ & 15 & $3.2 \mathrm{e} 5$ & $2.0 \mathrm{e} 5$ & $4.3 \mathrm{e} 5$ & $3.2 \mathrm{e} 5$ & & . & . & $\cdot$ & \\
\hline $1 e-3$ & 1 & $7.4 \mathrm{e} 7$ & $3.7 \mathrm{e} 7$ & $>7 \mathrm{e} 7$ & $4.1 \mathrm{e} 6$ & . & . & . & . & . \\
\hline & 0 & $10 e-3$ & $11 e-4$ & $22 e-3$ & $2.0 \mathrm{e} 6$ & & & & . & \\
\hline \multirow[t]{2}{*}{$1 e-8$} & . & . & . & . & & & . & 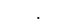 & . & \\
\hline & \multirow{2}{*}{\multicolumn{5}{|c|}{$f 130$ in $5-\mathrm{D}, \mathrm{N}=15, \mathrm{mFE}=5.00 \mathrm{e} 6$}} & \multirow{2}{*}{\multicolumn{5}{|c|}{$f 130$ in $20-\mathrm{D}, \mathrm{N}=15, \mathrm{mFE}=2.00 \mathrm{e} 7$}} \\
\hline$\Delta f$ & \# & & & & & \# & ERT & & & \\
\hline 10 & 15 & $1.7 \mathrm{e} 2$ & $1.3 \mathrm{e} 2$ & $2.2 \mathrm{e} 2$ & $1.7 \mathrm{e} 2$ & 0 & $25 e+0$ & $18 e+0$ & $29 e+0$ & $1.3 \mathrm{e} 7$ \\
\hline 1 & 15 & $2.5 \mathrm{e} 4$ & $1.7 \mathrm{e} 4$ & $3.2 \mathrm{e} 4$ & $2.5 \mathrm{e} 4$ & & . & . & . & . \\
\hline $1 e-1$ & 15 & $3.3 \mathrm{e} 5$ & $2.4 \mathrm{e} 5$ & $4.0 \mathrm{e} 5$ & 3. $3 \mathrm{e} 5$ & & . & r. & . & . \\
\hline $1 e-3$ & 1 & $7.4 \mathrm{e} 7$ & $2.5 \mathrm{e} 7$ & $>7 \mathrm{e} 7$ & $5.0 \mathrm{e} 6$ & & . & . & . & 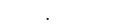 \\
\hline $1 e-5$ & 0 & $42 e-4$ & $16 e-4$ & $15 e-3$ & $2.5 \mathrm{e} 6$ & & . & . & . & . \\
\hline $1 e-8$ & 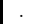 & & & 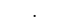 & & & & & . & \\
\hline
\end{tabular}

Table 2: Shown are, for functions $f_{121}-f_{130}$ and for a given target difference to the optimal function value $\Delta f$ : the number of successful trials (\#); the expected running time to surpass $f_{\mathrm{opt}}+\Delta f$ (ERT, see Figure 1); the $10 \%$-tile and $90 \%$-tile of the bootstrap distribution of ERT; the average number of function evaluations in successful trials or, if none was successful, as last entry the median number of function evaluations to reach the best function value $\left(\mathrm{RT}_{\text {succ }}\right)$. If $f_{\text {opt }}+\Delta f$ was never reached, figures in italics denote the best achieved $\Delta f$-value of the median trial and the $10 \%$ and $90 \%$-tile trial. Furthermore, $\mathbf{N}$ denotes the number of trials, and $\mathrm{mFE}$ denotes the maximum of number of function evaluations executed in one trial. See Figure 1 for the names of functions. 